\title{
Weed Management under Phosphorus Enrichment in Groundnut (Arachis hypogaea L.)
}

\author{
Harikesh Jat ${ }^{1}$, M.K. Kaushik ${ }^{2}$, S.K. Sharma ${ }^{3}$, V.K. Meena ${ }^{4}$
}

10.18805/LR-4624

\begin{abstract}
Background: Weeds infestation is one of the major threats to crop production and leads to severe crop-weed competition which finally reduce the crop productivity in term of quality and quantity. To enhance crop productivity therefore efficacy of various pre and post-emergence herbicides were evaluated on the basis of Amaranthus viridis, Commelina benghalensis and Cynodon dactylon population reduction, nutrient concentration and their uptake by groundnut.

Methods: The present investigation was conducted consecutively for two kharif seasons of 2016 and 2017, at Instructional Farm, CTAE, MPUAT, Udaipur with six weed management practices and four phosphorus levels thus, 30 treatment combinations; all were evaluated in split-plot design with three replications.

Result: The experimental results revealed that all herbicidal treatments as well as weed free recorded significantly lowest weed density as well as highest weed control efficiency, nitrogen, phosphorus and potassium concentration and also their uptake by groundnut as compared to weedy check except quizalofop-ethyl at 30 DAS in respect to control Cynodon dactylon. Further, pod yield response to phosphorus was found quadratic. The results also showed that phosphorus exhibit significantly increase nutrients concentration and their uptake by groundnut over control during the experimentation.
\end{abstract}

Key words: Groundnut, Nutrient concentration, Phosphorus, Phytotoxicity, Weed management.

\section{INTRODUCTION}

The oilseed production in India (89 million tonnes) lags behind the domestic demand and imported 148.20 million tonnes edible oil meet out total domestic demand of 235 million tonnes. Of this, around 5 million tonnes is exported or used in industries (IAS point, 2018). To bring the nation self- sufficient at oilseed front, the only option is to enhance the productivity since increase in acreage under oilseeds has limited or no scope in the country. On other hand, shrinking soil fertility during post green revolution era and heavy weed infestation during kharif season are another grave problem in achieving the sustainable crop production resulted in yield loss due to heavy weed infestation in groundnut ranged from $13-80 \%$ in India (Kumar et al., 2019). Due to intermittent rainfall during rainy season and scanty labour, manual weeding at right stage is difficult and time consuming and expensive, under this situation farmer adopt herbicidal use with suitable dose remains the pertinent choice for controlling weeds (Jadhav and Kashid, 2019).

India notified as the second largest producer of groundnut after China, occupies an important position in the Indian agricultural economy (APEDA, 2018). Arachis hypogaea $\mathrm{L}$. is self pollinated, unpredictable legume and adorned as the "wonder nut" commonly known as monkeynut, peanut and popularly called as moongphali belongs to the family fabaceae is largely grown as a small holding crop in rainfed area under arid and semi- arid conditions in the world (Hamakareem et al., 2016).

\section{${ }^{1}$ Govt. of Rajasthan, India}

${ }^{2}$ Department of Agromony, Rajasthan College of Agriculture, Maharana Pratap University of Agriculture and Technology, Udaipur313 001, Rajasthan, India.

${ }^{3}$ Directorate of Research, Maharana Pratap University of Agriculture and Technology, Udaipur-313 001, Rajasthan, India.

${ }^{4}$ ICAR- National Dairy Research Institute, Karnal-132 001, Haryana, India.

Corresponding Author: V.K. Meena, ICAR-National Dairy Research Institute, Karnal-132 001, Haryana, India.

Email: kumar.ladla@gmail.com

How to cite this article: Jat, H., Kaushik, M.K., Sharma, S.K. and Meena, V.K. (2021). Weed Management under Phosphorus Enrichment in Groundnut (Arachis hypogaea L.). Legume Research. DOI: $10.18805 /$ LR-4624.

Submitted: 31-03-2021 Accepted: 18-06-2021 Online: 01-09-2021

\section{MATERIALS AND METHOdS}

The present field experiment was carried out consecutively for two kharif seasons of 2016 and 2017, at Instructional Farm, MPUAT, Udaipur to examine effect of different weed management practices and phosphorus levels on weeds population, NPK concentration and uptake by groundnut. The experimental site is falls under agro-climatic zone IVa in SouthEastern region of Rajasthan, associated with typically semiarid and sub-tropical climate. The soil of experimental site was sandy loam in texture and slightly alkaline in reaction $(\mathrm{pH} 7.74)$, medium in available nitrogen $\left(261.10 \mathrm{~kg} \mathrm{ha}^{-1}\right)$, phosphorus (17.11 $\mathrm{kg} \mathrm{ha}^{-1}$ ) and high in available potassium 
(176.37 $\left.\mathrm{kg} \mathrm{ha}^{-1}\right)$. The experiments were laid out in split-plot design comprised six weed management practices viz., $\left(\mathrm{W}_{0}\right)$ weedy check, $\left(W_{1}\right)$ weed free (up to 60 DAS), $\left(W_{2}\right)$ pendimethalin $750 \mathrm{~g} \mathrm{ha}^{-1}$ and $\left(\mathrm{W}_{3}\right)$ oxyfluorfen $125 \mathrm{~g} \mathrm{ha}^{-1}$ as pre-emergence while, $\left(\mathrm{W}_{4}\right)$ imazethapyr $100 \mathrm{~g} \mathrm{ha}^{-1}$ and $\left(\mathrm{W}_{5}\right)$ quizalofop-ethyl $50 \mathrm{~g} \mathrm{ha}^{-1}$ as post-emergence (15 DAS) and five phosphorous levels i.e., $\left(P_{0}\right) 0$ control, $\left(P_{1}\right)$ 20, $\left(P_{2}\right) 40$, $\left(\mathrm{P}_{3}\right) 60$ and $\left(\mathrm{P}_{4}\right) 80 \mathrm{~kg} \mathrm{P}_{2} \mathrm{O}_{5}$ ha-1, thus making 30 treatment combinations, replicated thrice. In order to get well leveled pulverized soil for sowing, the experimental field was given one summer ploughing and 2 cross harrowing followed by planking to get good tilth. Healthy sound groundnut kernel treated with Carbendazim to prevent kernel borne disease followed by inoculation as per standard procedure with efficient strains Rhizobium leguminoserum kernels of test variety 'TG $37 \mathrm{~A}$ ' were sown at spacing of $30 \mathrm{~cm} \mathrm{X}$ $10 \mathrm{~cm}$ with a depth of nearly $4-5 \mathrm{~cm}$ by using seed rate of $100 \mathrm{~kg} \mathrm{ha}^{-1}$. Pre and post-emergence herbicides were applied at 2 and 15 DAS, respectively by using battery operated knapsack sprayer fitted with flat-fan nozzle with spray fluid of $500 \mathrm{~L} \mathrm{ha}^{-1}$ as well as in the designated plots the weeds were removed manually to keep weed free up to 60 DAS while, unweeded check plots were allowed to remain infested with weeds till harvesting of the crop. The recommended dose of nitrogen $30 \mathrm{~kg} \mathrm{ha}^{-1}$ and phosphorus (as per treatment) was applied as basal application through urea and DAP in the furrows below the kernel to all the plots. The rest of the packages of practices were adopted as per recommended in Rajasthan. Weeds density and weed control efficiency were recorded at 30, 45 DAS and harvest then weeds density data transformed to square root transformation $\sqrt{x+0.5}$ to normalize their distribution. Phytotoxicity observations were recorded on vein clearing, necrosis, wilting, epinasty and hyponasty etc. on $0-10$ scale at 7,14 and 21 days after spraying.

Representative samples of pod and haulm collected at harvest from each individual plots of experimental unit were oven dried at $65^{\circ} \mathrm{C}$ to a constant weight and grounded in laboratory mill. These samples were subjected to chemical analysis for determining $\mathrm{N}, \mathrm{P}$ and $\mathrm{K}$ concentration by using prescribed standard methods. The uptake of $\mathrm{N}, \mathrm{P}$ and $\mathrm{K}$ nutrient by pod and haulm were estimated by using the following formula.

Nutrient uptakeby pod/ haulm $\left(\mathrm{kg} \mathrm{ha}^{-1}\right)=$

$\frac{\text { Nutrient content in pod/ haulm }(\%) \times \mathrm{Pod} / \text { haulm yield }\left(\mathrm{kg} \mathrm{ha}^{-1}\right)}{100}$

The recorded data were analyzed using analysis of variance (ANOVA) technique (Gomez and Gomez 1984). The least significance test was used to decipher the main effects of treatments at $5 \%$ level of significance $(P<0.05)$.

\section{RESULTS AND DISCUSSION}

Across research study, in the experimental field weedy check plots, Arachis hypogaea L. was infested with mixed flora of broad-leaved and narrow-leaved weeds. Among the total weeds broad-leaved weeds (53\%) were more prominent as compared to narrow-leaved weeds (47\%). The weed flora under broad-leaved weeds includes many species mainly Amaranthus viridis (L.), Commelina benghalensis (L.), Digera arvensis Forsk, Trianthema portulacastrum (L.) and other broad-leaved weeds (Parthenium hysterophorus L. and Phyllanthus niruri Hook F.) whereas, Cynodon dactylon (L.), Cyperus rotundus (L.) and Echinochloa colonum (L.) were dominant species among narrow-leaved weeds (Fig 1 and 2).

\section{Individual weed density Amaranthus viridis}

Weed management treatments significantly reduced the density of Amaranthus viridis as against weedy check. Among herbicidal treatments, application of imazethapyr gave significant reduction of this weed count over other herbicides (Table 1). The magnitude of per cent decrease with imazethapyr (42.19, 55.92 and 55.92), oxyfluorfen (26.75, 43.00 and 50.79), pendimethalin (23.99, 38.06 and 49.07) and quizalofop-ethyl (13.69, 11.67 and 12.32) as compared to weedy check at 30,45 DAS and harvest, respectively.

\section{Commelina benghalensis}

Weed free up to 60 DAS was found significantly superior meanwhile, among herbicidal treatments imazethapyr was also significantly controlled this weed over rest of the herbicides. Further, application of imazethapyr and oxyfluorfen were equally efficient in term of reducing the density of Commelina benghalensis and found statistically at par with each other at 30 DAS.

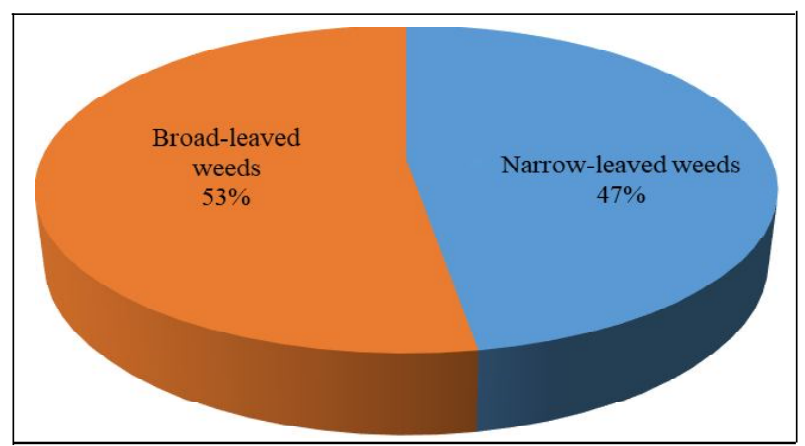

Fig 1: Weed flora in the experimental weedy check at harvest.

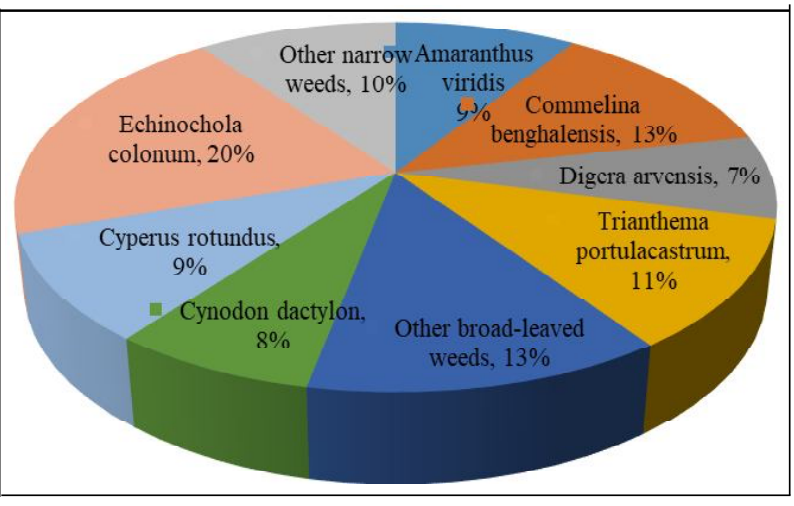

Fig 2: Weed flora in the experimental weedy check at harvest 


\section{Cynodon dactylon}

In comparison to weedy check, the herbicides in-question significantly reduced Cynodon dactylon density rather than quizalofop-ethyl. At harvest employed weed free up to 60 DAS, imazethapyr, pendimethalin, quizalofop-ethyl and oxyfluorfen accounted for $95.24,47.73,26.10,11.27$ and 9.12 per cent reduction in density of Cynodon dactylon over weedy check, respectively.

\section{Weed control efficiency}

It is manifested that highest broad - leaved weed control efficiency was recorded with imazethapyr followed by pendimethalin, oxyfluorfen and quizalofop-ethyl (Table 2). The highest weed control efficiency of narrow-leaved weeds at 30, 45 DAS and harvest was recorded under weed free followed by imazethapyr. The WCE of total weeds were the highest achieved by weed free followed by imazethapyr, pendimethalin and oxyfluorfen while, lowest was recorded under quizalofop-ethyl. It was due to lower weed population and total dry weight of weeds in these treatments due to better control of weeds. This study was supported by studies of Kumar and Chawla (2019) Singh et al. (2019) and Meena et al. (2020).

\section{Visual phytotoxicity scoring}

The imazethapyr application on groundnut elucidates slightly discoloration at 7 days after spray whereas, quizalofop-ethyl showed none of the phytotoxicity symptoms at 7, 14 and 21 days after spray (Table 3 a and b). Phytotoxicity to weeds indicates the degree of weed control whereas, phytotoxicity to crop indicates the degree of herbicide selectivity to crop and indicate whether it could safely be used in that crop or not.

\section{Optimum phosphorus dose}

Response of pod yield to varying levels of phosphorus was worked out and found quadratic. The functional form of yield response to phosphorus is given in (Fig 3). The economic optimum dose of phosphorus was computed as $62 \mathrm{~kg} \mathrm{ha}^{-1}$ with the corresponding pod yield of $1800 \mathrm{~kg} \mathrm{ha}^{-1}$.

Table 1: Effect of weed management practices and phosphorus levels on individual weed density (Pooled data of 2016 and 2017).

\begin{tabular}{|c|c|c|c|c|c|c|c|c|c|}
\hline \multirow{3}{*}{ Treatments } & \multicolumn{9}{|c|}{ Weed density (No. $\mathrm{m}^{-2}$ ) } \\
\hline & \multicolumn{3}{|c|}{ Amaranthus viridis } & \multicolumn{3}{|c|}{ Commelina benghalensis } & \multicolumn{3}{|c|}{ Cynodon dactylon } \\
\hline & 30 DAS & 45 DAS & Harvest & 30 DAS & 45 DAS & Harvest & 30 DAS & 45 DAS & Harvest \\
\hline \multicolumn{10}{|l|}{ Weed management practices } \\
\hline Weedy check & $\begin{array}{c}3.36 \\
(10.88)\end{array}$ & $\begin{array}{c}4.35 \\
(18.42)\end{array}$ & $\begin{array}{c}4.75 \\
(22.07)\end{array}$ & $\begin{array}{c}4.55 \\
(20.29)\end{array}$ & $\begin{array}{c}5.57 \\
(30.57)\end{array}$ & $\begin{array}{c}5.50 \\
(29.87)\end{array}$ & $\begin{array}{c}2.97 \\
(8.47)\end{array}$ & $\begin{array}{c}3.75 \\
(13.61)\end{array}$ & $\begin{array}{c}4.23 \\
(17.66)\end{array}$ \\
\hline Weed free (Up to 60 DAS) & $\begin{array}{c}0.71 \\
(0.00)\end{array}$ & $\begin{array}{c}0.71 \\
(0.00)\end{array}$ & $\begin{array}{c}1.59 \\
(2.02)\end{array}$ & $\begin{array}{c}0.71 \\
(0.00)\end{array}$ & $\begin{array}{c}0.71 \\
(0.00)\end{array}$ & $\begin{array}{c}1.12 \\
(0.77)\end{array}$ & $\begin{array}{c}0.71 \\
(0.00)\end{array}$ & $\begin{array}{c}0.71 \\
(0.00)\end{array}$ & $\begin{array}{c}1.09 \\
(0.84)\end{array}$ \\
\hline Pendimethalin $750 \mathrm{~g} \mathrm{ha}^{-1} \mathrm{PE}$ & $\begin{array}{c}2.96 \\
(8.27)\end{array}$ & $\begin{array}{c}3.45 \\
(11.41)\end{array}$ & $\begin{array}{c}3.42 \\
(11.24)\end{array}$ & $\begin{array}{c}2.75 \\
(7.09)\end{array}$ & $\begin{array}{c}4.36 \\
(18.59)\end{array}$ & $\begin{array}{c}4.56 \\
(20.32)\end{array}$ & $\begin{array}{c}2.64 \\
(6.55)\end{array}$ & $\begin{array}{c}3.32 \\
(10.57)\end{array}$ & $\begin{array}{c}3.68 \\
(13.05)\end{array}$ \\
\hline Oxyfluorfen $125 \mathrm{~g} \mathrm{ha}^{-1} \mathrm{PE}$ & $\begin{array}{c}2.90 \\
(7.97)\end{array}$ & $\begin{array}{c}3.31 \\
(10.50)\end{array}$ & $\begin{array}{c}3.35 \\
(10.86)\end{array}$ & $\begin{array}{c}2.52 \\
(5.84)\end{array}$ & $\begin{array}{c}4.55 \\
(20.22)\end{array}$ & $\begin{array}{c}4.44 \\
(19.28)\end{array}$ & $\begin{array}{c}2.55 \\
(6.05)\end{array}$ & $\begin{array}{c}3.54 \\
(12.07)\end{array}$ & $\begin{array}{c}4.07 \\
(16.05)\end{array}$ \\
\hline Imazethapyr $100 \mathrm{~g} \mathrm{ha}^{-1} \mathrm{PoE}$ & $\begin{array}{c}2.60 \\
(6.29)\end{array}$ & $\begin{array}{c}2.93 \\
(8.12)\end{array}$ & $\begin{array}{c}3.12 \\
(9.32)\end{array}$ & $\begin{array}{c}2.47 \\
(5.62)\end{array}$ & $\begin{array}{c}3.34 \\
(10.68)\end{array}$ & $\begin{array}{c}3.77 \\
(13.74)\end{array}$ & $\begin{array}{c}2.23 \\
(4.54)\end{array}$ & $\begin{array}{c}2.78 \\
(7.22)\end{array}$ & $\begin{array}{c}3.12 \\
(9.23)\end{array}$ \\
\hline Quizalofop-ethyl $50 \mathrm{~g} \mathrm{ha}^{-1} \mathrm{PoE}$ & $\begin{array}{c}3.14 \\
(9.39)\end{array}$ & $\begin{array}{c}4.09 \\
(16.27)\end{array}$ & $\begin{array}{c}4.45 \\
(19.35)\end{array}$ & $\begin{array}{c}4.08 \\
(16.19)\end{array}$ & $\begin{array}{c}5.04 \\
(24.90)\end{array}$ & $\begin{array}{c}5.29 \\
(27.56)\end{array}$ & $\begin{array}{c}2.93 \\
(8.15)\end{array}$ & $\begin{array}{c}3.58 \\
(12.33)\end{array}$ & $\begin{array}{c}4.02 \\
(15.67)\end{array}$ \\
\hline SEm \pm & 0.02 & 0.01 & 0.02 & 0.03 & 0.02 & 0.02 & 0.04 & 0.02 & 0.03 \\
\hline $\operatorname{LSD}(P=0.05)$ & 0.06 & 0.05 & 0.07 & 0.08 & 0.06 & 0.07 & 0.12 & 0.07 & 0.10 \\
\hline Phosphorus levels $\left(\mathrm{P}_{2} \mathrm{O}_{5} \mathrm{~kg}\right.$ ha & & & & & & & & & \\
\hline 0 (Control) & $\begin{array}{c}2.60 \\
(7.04)\end{array}$ & $\begin{array}{c}3.13 \\
(10.69)\end{array}$ & $\begin{array}{c}3.43 \\
(12.41)\end{array}$ & $\begin{array}{c}2.83 \\
(9.05)\end{array}$ & $\begin{array}{c}3.91 \\
(17.37)\end{array}$ & $\begin{array}{c}4.11 \\
(18.61)\end{array}$ & $\begin{array}{c}2.32 \\
(5.55)\end{array}$ & $\begin{array}{c}2.92 \\
(9.17)\end{array}$ & $\begin{array}{c}3.36 \\
(12.07)\end{array}$ \\
\hline 20 & $\begin{array}{c}2.61 \\
(7.10)\end{array}$ & $\begin{array}{c}3.13 \\
(10.73)\end{array}$ & $\begin{array}{c}3.44 \\
(12.47)\end{array}$ & $\begin{array}{c}2.84 \\
(9.10)\end{array}$ & $\begin{array}{c}3.92 \\
(17.42)\end{array}$ & $\begin{array}{c}4.11 \\
(18.63)\end{array}$ & $\begin{array}{c}2.33 \\
(5.59)\end{array}$ & $\begin{array}{c}2.93 \\
(9.20)\end{array}$ & $\begin{array}{c}3.36 \\
(12.00)\end{array}$ \\
\hline 40 & $\begin{array}{c}2.61 \\
(7.12)\end{array}$ & $\begin{array}{c}3.14 \\
(10.77)\end{array}$ & $\begin{array}{c}3.44 \\
(12.46)\end{array}$ & $\begin{array}{c}2.84 \\
(9.15)\end{array}$ & $\begin{array}{c}3.93 \\
(17.48)\end{array}$ & $\begin{array}{c}4.11 \\
(18.60)\end{array}$ & $\begin{array}{c}2.34 \\
(5.63)\end{array}$ & $\begin{array}{c}2.94 \\
(9.29)\end{array}$ & $\begin{array}{c}3.36 \\
(12.06)\end{array}$ \\
\hline 60 & $\begin{array}{c}2.62 \\
(7.16)\end{array}$ & $\begin{array}{c}3.14 \\
(10.82)\end{array}$ & $\begin{array}{c}3.45 \\
(12.53)\end{array}$ & $\begin{array}{c}2.85 \\
(9.21)\end{array}$ & $\begin{array}{c}3.93 \\
(17.54)\end{array}$ & $\begin{array}{c}4.12 \\
(18.66)\end{array}$ & $\begin{array}{c}2.34 \\
(5.65)\end{array}$ & $\begin{array}{c}2.96 \\
(9.38)\end{array}$ & $\begin{array}{c}3.39 \\
(12.21)\end{array}$ \\
\hline 80 & $\begin{array}{c}2.63 \\
(7.24)\end{array}$ & $\begin{array}{c}3.16 \\
(10.92)\end{array}$ & $\begin{array}{c}3.46 \\
(12.53)\end{array}$ & $\begin{array}{c}2.87 \\
(9.33)\end{array}$ & $\begin{array}{c}3.95 \\
(17.66)\end{array}$ & $\begin{array}{c}4.11 \\
(18.47)\end{array}$ & $\begin{array}{c}2.36 \\
(5.71)\end{array}$ & $\begin{array}{c}2.97 \\
(9.45)\end{array}$ & $\begin{array}{c}3.37 \\
(12.06)\end{array}$ \\
\hline SEm \pm & 0.01 & 0.01 & 0.02 & 0.01 & 0.01 & 0.02 & 0.01 & 0.01 & 0.01 \\
\hline LSD $(P=0.05)$ & NS & NS & NS & NS & NS & NS & NS & NS & NS \\
\hline
\end{tabular}

*Data subjected to $\sqrt{x+0.5}$ transformation and figures in parenthesis are original weed count $\mathrm{m}^{-2}$. 
Weed Management under Phosphorus Enrichment in Groundnut (Arachis hypogaea L.)

\section{Nutrient concentration}

Under investigation the significant maximum N (3.570 and $1.827 \%), P(0.810$ and $0.496 \%)$ and $K(0.768$ and $1.241 \%)$ concentrations in pod and haulm were registered under weed free over oxyfluorfen, quizalofop-ethyl and weedy check but, found statistically at par with imazethapyr and pendimethalin in respect to $\mathrm{N}$ and $\mathrm{K}$ concentration in pod whereas, $\mathrm{N}$ and $\mathrm{P}$ concentration in halum as well as $\mathrm{P}$ concentration in haulm and also found statistically at par with only pendimethalin in respect to $\mathrm{N}$ and $\mathrm{P}$ concentration in halum and pod, respectively (Table 4). This might be due to weed management practices clearly attributed to

Table 2: Effect of weed management practices on weed control efficiency (Pooled data of 2016 and 2017).

\begin{tabular}{|c|c|c|c|c|c|c|c|c|c|}
\hline \multirow{3}{*}{ Treatments } & \multicolumn{9}{|c|}{ Weed control efficiency (\%) } \\
\hline & \multicolumn{3}{|c|}{ Broad-leaved weeds } & \multicolumn{3}{|c|}{ Narrow-leaved weeds } & \multicolumn{3}{|c|}{ Total weeds } \\
\hline & 30 DAS & 45 DAS & Harvest & 30 DAS & 45 DAS & Harvest & 30 DAS & 45 DAS & Harvest \\
\hline \multicolumn{10}{|l|}{ Weed management practices } \\
\hline Weedy check & 0.00 & 0.00 & 0.00 & 0.00 & 0.00 & 0.00 & 0.00 & 0.00 & 0.00 \\
\hline Weed free (Up to 60 DAS) & 100.00 & 100.00 & 96.18 & 100.00 & 100.00 & 92.25 & 100.00 & 100.00 & 94.46 \\
\hline Pendimethalin $750 \mathrm{~g} \mathrm{ha}^{-1} \mathrm{PE}$ & 67.00 & 60.35 & 45.45 & 75.02 & 66.98 & 49.24 & 69.70 & 62.86 & 47.11 \\
\hline Oxyfluorfen $125 \mathrm{~g} \mathrm{ha}^{-1} \mathrm{PE}$ & 69.51 & 63.89 & 48.54 & 59.32 & 51.74 & 36.43 & 66.08 & 59.28 & 43.23 \\
\hline Imazethapyr $100 \mathrm{~g} \mathrm{ha}^{-1} \mathrm{PoE}$ & 76.08 & 69.20 & 60.57 & 67.29 & 62.29 & 56.73 & 73.13 & 66.58 & 58.88 \\
\hline Quizalofop-ethyl $50 \mathrm{~g} \mathrm{ha}^{-1} \mathrm{PoE}$ & 18.07 & 16.02 & 12.08 & 69.61 & 62.18 & 49.68 & 35.39 & 33.52 & 28.57 \\
\hline
\end{tabular}

Table 3 (a): Visual observation on phytotoxicity of post-emergence herbicides on groundnut.

\begin{tabular}{|c|c|c|c|c|c|c|c|c|c|}
\hline \multirow{2}{*}{ Treatments } & \multicolumn{3}{|c|}{ Chlorosis score $\left(\mathrm{DAS}^{*}\right)$} & \multicolumn{3}{|c|}{ Necrosis score (DAS) } & \multicolumn{3}{|c|}{ Wilting score (DAS) } \\
\hline & 7 & 14 & 21 & 7 & 14 & 21 & 7 & 14 & 21 \\
\hline Imazethapyr $100 \mathrm{~g} \mathrm{ha}^{-1} \mathrm{PoE}$ & 1 & 0 & 0 & 0 & 0 & 0 & 0 & 0 & 0 \\
\hline Quizalofop-ethyl $50 \mathrm{~g} \mathrm{ha}^{-1} \mathrm{PoE}$ & 0 & 0 & 0 & 0 & 0 & 0 & 0 & 0 & 0 \\
\hline Weed free & 0 & 0 & 0 & 0 & 0 & 0 & 0 & 0 & 0 \\
\hline
\end{tabular}

Table 3 (b): Visual observation on phytotoxicity of post-emergence herbicides on groundnut.

\begin{tabular}{|c|c|c|c|c|c|c|c|c|c|}
\hline \multirow{2}{*}{ Treatments } & \multicolumn{3}{|c|}{ Scorching score (DAS) } & \multicolumn{3}{|c|}{ Hyponasty score (DAS) } & \multicolumn{3}{|c|}{ Epinasty score (DAS) } \\
\hline & 7 & 14 & 21 & 7 & 14 & 21 & 7 & 14 & 21 \\
\hline Imazethapyr $100 \mathrm{~g} \mathrm{ha}^{-1} \mathrm{PoE}$ & 0 & 0 & 0 & 0 & 0 & 0 & 0 & 0 & 0 \\
\hline Quizalofop-ethyl $50 \mathrm{~g} \mathrm{ha}^{-1} \mathrm{PoE}$ & 0 & 0 & 0 & 0 & 0 & 0 & 0 & 0 & 0 \\
\hline Weed free & 0 & 0 & 0 & 0 & 0 & 0 & 0 & 0 & 0 \\
\hline
\end{tabular}

* Days after post-emergence herbicide spray.

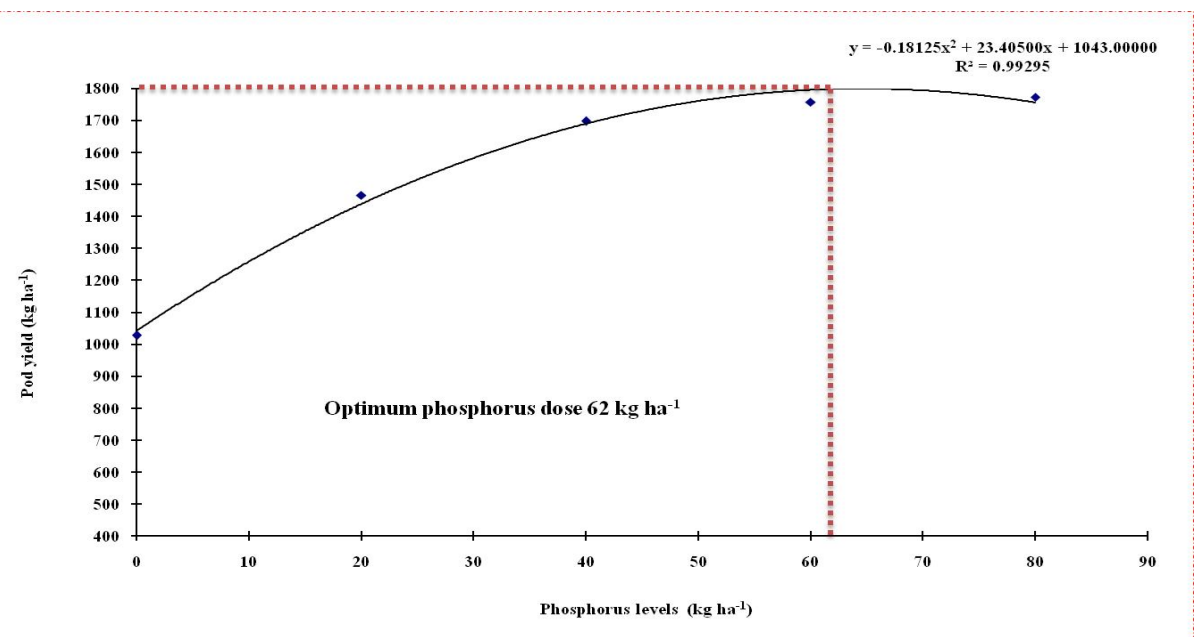

Fig 3: Response curve for optimum phosphorus dose and pod yield. 
Weed Management under Phosphorus Enrichment in Groundnut (Arachis hypogaea L.)

the reduction in interference of the weeds which ultimately favored better growth environment for the crop. Thus, under least crop weed competition, adequate availability of light, temperature and space along with moisture and nutrients, improved nutrients concentration in plant.

The groundnut crop under the influence of $60 \mathrm{~kg} \mathrm{P}{ }_{2} \mathrm{O}_{5}$ $\mathrm{ha}^{-1}$ accounted significantly greater $\mathrm{P}$ concentration in pod while, significantly higher $\mathrm{N}$ in both pod and haulm as well as $\mathrm{P}$ in haulm recorded under $80 \mathrm{~kg} \mathrm{P}_{2} \mathrm{O}_{5}$ ha $^{-1}$ over preceding lower levels. Whereas, $\mathrm{K}$ did not significantly influence by phosphorus application. It might be due to improvement in nutritional status of plant under application of phosphorus seems to be primarily on account of better growth of roots, which might have increased the absorption and efficient translocation of these towards plant system. The marked increment of $\mathrm{N}, \mathrm{P}$ and $\mathrm{K}$ concentration in plant parts with the application of increased phosphorus seems to be on account of its pivotal role in the formation of roots, their proliferation, microbial population and activity in the soil. (Meena et al. 2016; Dutta et al. 2020).

Table 4: Effect of weed management practices and phosphorus levels on nutrient concentration in groundnut (Pooled data of 2016 and 2017).

\begin{tabular}{|c|c|c|c|c|c|c|}
\hline \multirow{2}{*}{ Treatments } & \multicolumn{2}{|c|}{ Nitrogen (\%) } & \multicolumn{2}{|c|}{ Phosphorus (\%) } & \multicolumn{2}{|c|}{ Potassium (\%) } \\
\hline & Pod & Haulm & Pod & Haulm & Pod & Haulm \\
\hline \multicolumn{7}{|l|}{ Weed management practices } \\
\hline Weedy check & 3.424 & 1.723 & 0.729 & 0.447 & 0.739 & 1.225 \\
\hline Weed free (Up to 60 DAS) & 3.570 & 1.827 & 0.810 & 0.496 & 0.768 & 1.241 \\
\hline Pendimethalin $750 \mathrm{~g} \mathrm{ha}^{-1} \mathrm{PE}$ & 3.561 & 1.817 & 0.797 & 0.492 & 0.762 & 1.233 \\
\hline Oxyfluorfen $125 \mathrm{~g} \mathrm{ha}^{-1} \mathrm{PE}$ & 3.493 & 1.796 & 0.791 & 0.485 & 0.759 & 1.227 \\
\hline Imazethapyr $100 \mathrm{~g} \mathrm{ha}^{-1} \mathrm{PoE}$ & 3.569 & 1.808 & 0.807 & 0.494 & 0.765 & 1.233 \\
\hline Quizalofop-ethyl $50 \mathrm{~g} \mathrm{ha}^{-1} \mathrm{PoE}$ & 3.518 & 1.802 & 0.781 & 0.482 & 0.756 & 1.231 \\
\hline $\mathrm{SEm} \pm$ & 0.017 & 0.006 & 0.005 & 0.002 & 0.002 & 0.008 \\
\hline $\operatorname{LSD}(P=0.05)$ & 0.051 & 0.017 & 0.015 & 0.005 & 0.007 & NS \\
\hline \multicolumn{7}{|l|}{ Phosphorus levels $\left(\mathrm{P}_{2} \mathrm{O}_{5} \mathrm{~kg} \mathrm{ha}^{-1}\right)$} \\
\hline 0 (Control) & 3.364 & 1.549 & 0.722 & 0.426 & 0.755 & 1.226 \\
\hline 20 & 3.486 & 1.795 & 0.773 & 0.467 & 0.756 & 1.228 \\
\hline 40 & 3.570 & 1.857 & 0.793 & 0.491 & 0.758 & 1.229 \\
\hline 60 & 3.576 & 1.878 & 0.817 & 0.512 & 0.759 & 1.236 \\
\hline 80 & 3.616 & 1.898 & 0.824 & 0.517 & 0.763 & 1.238 \\
\hline $\mathrm{SEm} \pm$ & 0.012 & 0.003 & 0.003 & 0.001 & 0.002 & 0.004 \\
\hline $\operatorname{LSD}(P=0.05)$ & 0.035 & 0.007 & 0.009 & 0.003 & NS & NS \\
\hline
\end{tabular}

Table 5: Effect of weed management practices and phosphorus levels on nutrient uptake by groundnut (Pooled data of 2016 and 2017 ).

\begin{tabular}{|c|c|c|c|c|c|c|}
\hline \multirow{2}{*}{ Treatments } & \multicolumn{2}{|c|}{ Nitrogen $\left(\mathrm{kg} \mathrm{ha}^{-1}\right)$} & \multicolumn{2}{|c|}{ Phosphorus $\left(\mathrm{kg} \mathrm{ha}^{-1}\right)$} & \multicolumn{2}{|c|}{ Potassium (kg ha-1) } \\
\hline & Pod & Haulm & Pod & Haulm & Pod & Haulm \\
\hline \multicolumn{7}{|l|}{ Weed management practices } \\
\hline Weedy check & 33.24 & 38.78 & 7.12 & 10.09 & 7.13 & 27.35 \\
\hline Weed free (Up to 60 DAS) & 64.81 & 56.79 & 14.79 & 15.28 & 13.89 & 37.94 \\
\hline Pendimethalin $750 \mathrm{~g} \mathrm{ha}^{-1} \mathrm{PE}$ & 62.38 & 54.37 & 14.01 & 14.77 & 13.28 & 36.72 \\
\hline Oxyfluorfen $125 \mathrm{~g} \mathrm{ha}^{-1} \mathrm{PE}$ & 53.65 & 51.64 & 12.19 & 13.89 & 11.60 & 34.80 \\
\hline Imazethapyr $100 \mathrm{~g} \mathrm{ha}^{-1} \mathrm{PoE}$ & 62.95 & 54.23 & 14.29 & 14.91 & 13.50 & 36.75 \\
\hline Quizalofop-ethyl $50 \mathrm{~g} \mathrm{ha}^{-1} \mathrm{PoE}$ & 51.72 & 52.06 & 11.50 & 13.89 & 11.07 & 35.20 \\
\hline $\mathrm{SEm} \pm$ & 0.84 & 0.60 & 0.19 & 0.17 & 0.16 & 0.47 \\
\hline $\operatorname{LSD}(P=0.05)$ & 2.60 & 1.85 & 0.58 & 0.52 & 0.50 & 1.44 \\
\hline \multicolumn{7}{|l|}{ Phosphorus levels $\left(\mathrm{P}_{2} \mathrm{O}_{5} \mathrm{~kg} \mathrm{ha}^{-1}\right)$} \\
\hline 0 (Control) & 34.65 & 31.19 & 7.47 & 8.61 & 7.75 & 24.68 \\
\hline 20 & 51.28 & 51.03 & 11.40 & 13.32 & 11.11 & 34.88 \\
\hline 40 & 60.81 & 56.38 & 13.54 & 14.95 & 12.90 & 37.29 \\
\hline 60 & 63.00 & 58.51 & 14.46 & 15.95 & 13.39 & 38.45 \\
\hline 80 & 64.23 & 59.44 & 14.70 & 16.19 & 13.56 & 38.66 \\
\hline SEm \pm & 0.39 & 0.21 & 0.09 & 0.06 & 0.07 & 0.16 \\
\hline $\operatorname{LSD}(P=0.05)$ & 1.09 & 0.59 & 0.25 & 0.17 & 0.21 & 0.45 \\
\hline
\end{tabular}




\section{Nutrient uptake}

All the weed management practices significantly influence the $\mathrm{N}, \mathrm{P}$ and $\mathrm{K}$ uptake by pod and haulm and being maximum recorded under weed free over rest of the treatments (Table 5). Which might be ascribed to higher yield under these treatments as uptake of nutrient is mainly the function of crop yield and nutrient concentration. Thus, higher nutrient uptake by crop might be due to decreased crop weed competition concurrently increased nutrient availability, better crop growth and higher crop biomass production coupled with more nutrient content. Results of present investigation corroborate with the finding of Kumbar et al. (2014) and Samant and Mishra (2014).

Application of $80 \mathrm{~kg} \mathrm{P}_{2} \mathrm{O}_{5}$ ha $^{-1}$ resulted in significantly higher $\mathrm{N}$ uptake by pod and haulm as well as $\mathrm{P}$ uptake by haulm. Whereas, significantly greater $P$ uptake by pod, $K$ uptake by pod and haulm were noticed with $60 \mathrm{~kg} \mathrm{P}_{2} \mathrm{O}_{5}$ ha ${ }^{1}$ over preceding phosphorus levels. Thus, higher nutrient uptake by crop might be due to the nutrient accumulation is dependent on concentration at the cellular level and dry matter accumulation. Hence, improvement in these components under phosphorus application reflected in higher uptake of nutrients by the crops. Hadwani and Gundalia (2005) and Dutta et al. (2020).

\section{REFERENCES}

APEDA, (2018). Groundnut_survey_report. Kharif-2018 Survey of Groundnut Crop. pp-1.

Dutta, S., Singh, M., Meena, R.K., Onte, S., Basak, N., Kumar, S. and Meena, V.K. (2020). Effect of organic and inorganic nutrient sources on growth, yield, nutrient uptake and economics of fodder cowpea [Vigna unguiculata (L.) Walp.]. Legume Research. 10.18805/LR-4181.

Gomez, K.A. and Gomez, A.A. (1984). Statistical Procedures for Agricultural Research, (2 ${ }^{\text {nd }}$ Ed.) John Willey and Sons, Singapore.

Hadwani, G.J. and Gundalia, J.D. (2005). Effect of N.P and K. levels on yield, nutrient content, uptake and quality of summer groundnut grown on Typic Haplustepts. Journal of the Indian Society of Soil Science. 53: 125-128.
Hamakareem, H.F., Hamahasan, B.M. and Ali, S.H.S. (2016). Influence of plant spacing on the growth and yield of groundnut (Arachis hypogaea L.). International Journal of Current Research in Biosciences and Plant Biology. 3: 7-12.

IAS Point. (2018). Oil seeds: area, production and issues, https:// www.gktoday.in/ academy/article/current-data-on-oilseeds-production-in-India.

Jadhav, V.T. and Kashid, N.V. (2019). Integrated weed management in soybean. Indian Journal of Weed Science. 51: 81-82.

Kumar, B.N., Subramanyam, D., Nagavani, A.V. and Umamahesh, V. (2019). Weed management in groundnut with new herbicide molecules. Indian Journal of Weed Science. 51: 306-307.

Kumar, M. and Chawla, J.S. (2019). Comparative study on weed control efficacy of different pre-and post-emergence herbicides in Kharif maize. Indian Journal of Weed Science. 51: 32-35.

Kumbar, B., Prasad, T.V.R., Sanjay, M.T., Mallikarjuna, G.B., Hatti, V. and Madhukumar, V. (2014). Influence of weed management practices on crop growth, nutrient uptake and yield of groundnut under irrigated condition. The Bioscan. 9: 1843-1846.

Meena, O.P., Yadav, M.R., Kumar, V., Goyal, S.K., Meena, A.K., Yadav, H.L. and Meena, V.K. (2020). Effect of different weed management practices on weed dynamics, productivity and farm profitability of cluster bean. Legume Research. 10.18805/LR-4303.

Meena, V.K., Kaushik, M.K., Kumar, R., Singh, M., Meena, B.L., Meena, B.P., Meena, R.K., Kumar U. and Kumar, S. (2016). Influence of growth regulators on nutrient concentrations, nutrient uptake and quality parameters of cluster bean varieties. Legume Research. 39: 797801.

Samant, T.K. and Mishra, K.N. (2014). Efficacy of post-emergence herbicide quizalofop-ethyl for controlling grassy weeds in groundnut. Indian Journal of Agricultural Research. 48: 488-492.

Singh, S.P., Yadav, R.S., Godara, S.L., Kumawat, A. and Birbal. (2019). Herbicidal weed management in groundnut (Arachis hypogaea L.). Legume Research. 42: 829-833. 